\title{
MucR and MucS Activate exp Genes Transcription and Galactoglucan Production in Sinorhizobium meliloti EFB1
}

\author{
Javier Lloret, Marta Martín, Roke I. Oruezabal, Ildefonso Bonilla, and Rafael Rivilla \\ Departamento de Biología, Universidad Autónoma de Madrid, Campus de Cantoblanco, 2049 Madrid, Spain
}

Submitted 20 February 2001. Accepted 7 September 2001.

\begin{abstract}
When grown under standard conditions, Sinorhizobium meliloti EFB1 simultaneously produces two acidic exopolysaccharides, succinoglycan and galactoglucan, yielding very mucoid colonies. In this strain, MucR is essential for galactoglucan synthesis. A mutation in the mucS gene resulted in less mucoid colonies than in the wild-type EFB1. This mucS $^{-}$strain was complemented to the wild-type phenotype by the cloned mucS gene, indicating that mucS is necessary for a wild-type level of galactoglucan production. Reverse transcription-polymerase chain reaction analysis of $\exp$ genes, which encode the pathway for galactoglucan production, in EFB1 and in the mutants affected in mucS, $m u c R$, and both genes simultaneously, showed that MucS is a transcriptional activator of the $\exp$ genes but does not affect its own transcription. Furthermore, MucR is necessary for mucS transcriptional activation. As introduction of a cloned mucS gene in a $m u c R^{-}$strain yielded colonies less mucoid than the wild type, MucR could also activate exp genes transcription through other pathways. Deletion analysis of the $\exp E$ promoter showed a region important for transcription and MucS activation. This region, containing a palindrome, is present in the putative $\exp A, \exp C, \operatorname{expD}$, and $\exp E$ promoters but not in the mucS promoter, suggesting that it is the target for MucS. A mucR ${ }^{-}$mucS ${ }^{-}$mutant, which does not produce galactoglucan, was impaired in competitive nodulation of alfalfa in soil microcosms, indicating another possible role for this exopolysaccharide in symbiosis.
\end{abstract}

Sinorhizobium meliloti can produce two types of acidic exopolysaccharides, succinoglycan and galactoglucan. Succinoglycan production has been shown to be a necessary trait for nodule invasion and, therefore, for successful nitrogen-fixing symbiosis (Battisti et al. 1992; Gonzalez et al. 1996), although at least in symbiosis with alfalfa, it can be replaced by galactoglucan or capsular polysaccharide production (Glazebrook and Walker 1989; Leigh and Walker 1994). Galactoglucan production requires the expression of the $\exp$ genes that encode

Corresponding author: R. Rivilla; Telephone: +34-91-3978188; E-mail: rafael.rivilla@uam.es.

Current address of J. Lloret: Department of Biology, Massachusetts Institute of Technology, Boston, MA, U. S. A.

J. Lloret and M. Martín contributed equally to this work and are joint first authors. the enzymes required for production of this exopolysaccharide and are clustered in the second megaplasmid (pSymb) (Astete and Leigh 1996). Regulation of galactoglucan production is complex and influenced by environmental factors such as cell density and salt and phosphate concentrations (Astete and Leigh 1996; Lloret et al. 1998; Rüberg et al. 1999). There is also a balance between galactoglucan and succinoglycan produced under each phosphate concentration (Mendrygal and Gonzalez 2000), indicating a coordinated regulation of the production of both exopolysaccharides.

Three loci implicated in regulating galactoglucan production have been identified, $\exp R, m u c R$, and mucS (also called $\exp G)$, although their precise roles have not been established (Glazebrook and Walker 1989; Keller et al. 1995; Martin et al. 2000). MucR is a zinc-finger protein encoded by a chromosomal gene (Keller et al. 1995) that, in strains such as Rm2011 that normally do not produce galactoglucan, acts as a repressor for the synthesis of the exopolysaccharide (Bertram-Drogatz et al. 1998; Keller et al. 1995). In strain Rm2011, the mucR mutant does produce galactoglucan, although only the high-molecular-weight-fraction (Gonzalez et al. 1996). However, in strain EFB1, which produces high amounts of galactoglucan showing a very mucoid phenotype, MucR is necessary for galactoglucan production (Martin et al. 2000). MucS is a helixturn-helix protein encoded by a gene within the exp regulon. In $\mathrm{Rm} 2011$, MucS is required for the activation of the expression of at least one gene of the $\exp E$ complementation group under low phosphate concentrations (Astete and Leigh 1996). In strain EFB1, a mucS mutant showed a 95\% reduction in the expression of the $\exp E$ promoter (Martin et al. 2000). However, as two different mutations within the gene coding region showed different phenotypes in the Rm2011 background (Becker et al. 1997), its role in galactoglucan production and exp genes activation has not been established. Furthermore, Rüberg and associates (1999) found that the deletion of the $m u c S$ gene had no influence on the level of exp genes expression in a Rm2011 mucR mutant.

Because $S$. meliloti EFB1 synthesizes galactoglucan under standard growth conditions, it is an adequate strain to study galactoglucan production (Lloret et al. 1998; Martin et al. 2000). This study analyzes the role played by the regulatory genes $m u c R$ and $m u c S$ in the production of galactoglucan by $S$. meliloti EFB1 and the possible role of this exopolysaccharide in symbiosis. We have studied the exp genes transcription level in different EFB1 backgrounds, wild type, a mucS mutant, a mucR mutant, and a double mucRmucS mutant. We have also analyzed the $\exp E$ promoter by nested deletions to find regions implicated in its regulation. 


\section{RESULTS AND DISCUSSION}

$m u c S$ is essential for wild-type levels of galactoglucan production in EFB1.

We have previously shown that mucR is essential for galactoglucan production in S. meliloti EFB1 (Martín et al. 2000), as a $m u c R$ mutant is completely nonmucoid and produces colonies indistinguishable from the colonies produced by a mutant carrying a 3.8-kb deletion affecting $\operatorname{expD}, m u c S$, and $\exp C$. In order to investigate the role played by mucS in exp genes regulation and galactoglucan production, an EFB1 mucS deletion mutant (EFB207) was constructed by eliminating an internal XhoI fragment from a pLAFR3-cloned mucS gene (Fig. 1) followed by homologous recombination in an EFB1 wild type. Polymerase chain reaction (PCR) and Southern blotting (data not shown) confirmed the absence of a complete mucS gene in EFB207. This deletion eliminates most of the MucS open reading frame (ORF), including the helix-turn-helix motif. EFB207 produces a lower amount of galactoglucan than does EFB1 as judged by the less-mucoid colony morphology on tryptone- yeast extract (TY) plates. These results indicate that mucS is necessary for the full production of galactoglucan, although it is not essential for the production of this exopolysaccharide. The EFB207 mutant was complemented to the wild-type mucoid phenotype by the cosmid pBG1000 (data not shown), which contains mucS and other exp genes. Furthermore, the plasmid pBG1140, which contains only the mucS gene, also complemented the mutation (Fig. 2C), while a similar construct, pBG1141, containing the mucS gene with the internal XhoI fragment deleted, did not (Fig. 2D). These results demonstrate the essentiality of a functional $m u c S$ gene for wild-type levels of galactoglucan production in S. meliloti EFB1.

\section{MucS is a transcriptional activator of the exp promoters and is not autoregulated.}

To investigate the transcriptional activation role that MucS and MucR play, we have analyzed the transcription level of all the exp promoters in the wild-type and mutant backgrounds. Transcription was analyzed by reverse-transcription PCR (RTPCR) with total RNA extracted from strains EFB1, EFB207
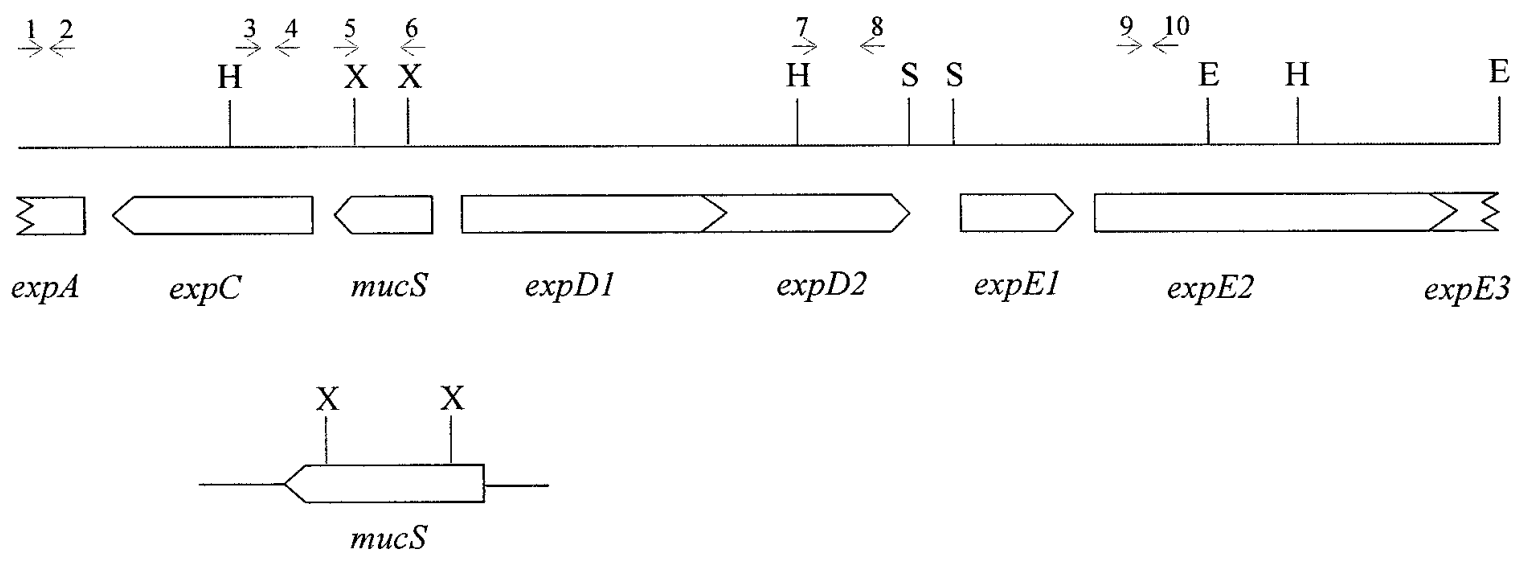

pBG1140 (pLAFR3); pBG1228 (pML122)
pBG1141 (pLAFR3)

Fig. 1. Physical and genetic map of part of the exp regulon in Sinorhizobium meliloti EFB1. Arrows indicate the primers used for reverse-transcriptionpolymerase chain reaction experiments and are numbered according to Table 1 . The $m u c S$-containing plasmids are indicated, with the vector used in parentheses. Only relevant restriction sites are shown. $\mathrm{E}=E c o \mathrm{RI}, \mathrm{H}=H$ indIII, $\mathrm{S}=S p h \mathrm{I}$, and $\mathrm{X}=\mathrm{Xho \textrm {I }}$.

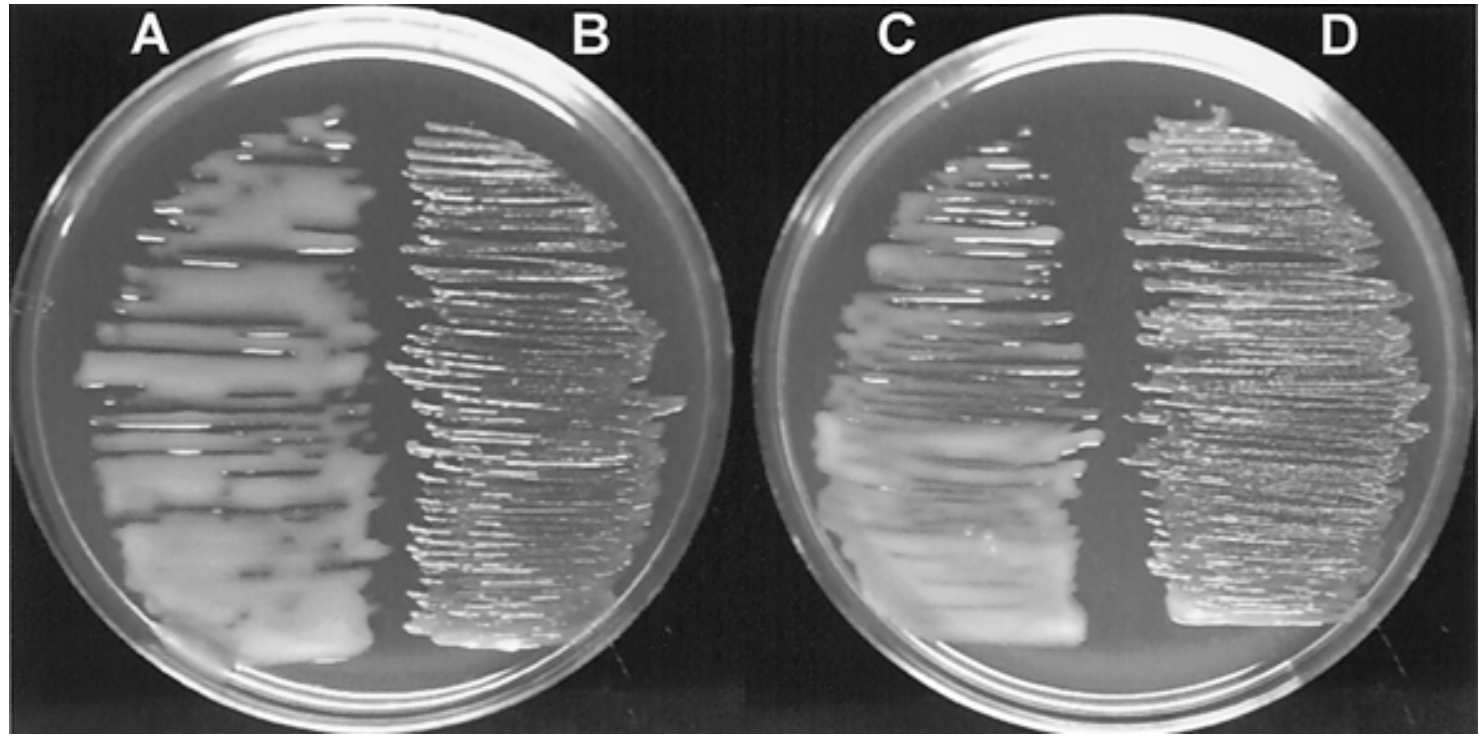

Fig. 2. Complementation analysis of the mucS mutant EFB207. A, EFB1 (wild type). B, EFB207 (mucS-). C, EFB207 pBG1140. D, EFB207 pBG1141. 
$\left(m u c S^{-}\right)$, EFB208 $\left(m u c R^{-}\right)$, and EFB209 $\left(m u c R^{-} m u c S^{-}\right)$, using primers designed from the sequence of an ORF in each exp operon (Table 1, Fig. 1). Double mutant EFB209 was constructed by cloning an internal fragment of the $m u c R$ gene (Martin et al. 2000 ) in suicide vector pK18mobsac followed by homologous recombination in the EFB207 background.

As shown in Figure 3, the transcript levels of $\operatorname{expA,} \operatorname{expC}$, $\exp D$, and $\exp E$ were higher in EFB1 than in EFB207, indicating that MucS is activating the transcription of these $\exp$ genes. However, the mucS transcript was expressed at the same level in both EFB1 and EFB207, showing that the mucS promoter does not require MucS for its transcription, and therefore, it is not autoregulated. There was no transcript from $m u c S$ in $m u c R^{-}$ and $m u c R^{-} m u c S^{-}$backgrounds, indicating that MucR is necessary for $m u c S$ transcription. Although galactoglucan production regulation is complex and influenced by different environmental factors, our results show that, in S. meliloti EFB1, MucR is regulating the activation of the mucS gene, which encodes a transcriptional activator required for the exp genes expression and for the production of galactoglucan observed under normal growth conditions. It is interesting to note that mutants affected in mucR homologues in Agrobacterium tumefaciens (Cooley et al. 1991) and Rhizobium etli (Bittinger and Handelsman 2000) also show a nonmucoid phenotype.
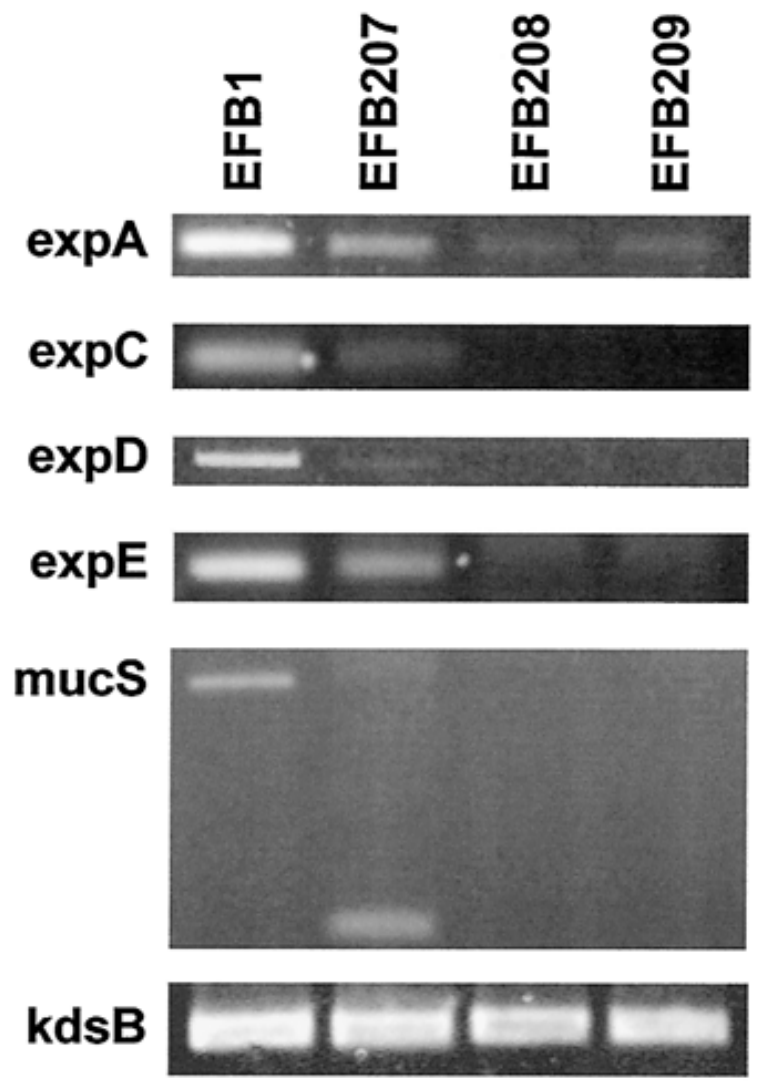

Fig. 3. Reverse-transcription-polymerase chain reaction (RT-PCR) analysis of the exp operons and mucS in EFB1 and mutant backgrounds; EFB1 (wild type), EFB207 (muc $\left.S^{-}\right)$, EFB208 $\left(m u c R^{-}\right)$, and EFB209 (muc $\mathrm{S}^{-}$mис $^{-}$). Total RNA was extracted, and $100 \mathrm{pg}$ were subjected to RT-PCR. The $k d s B$ gene was subjected to RT-PCR as a positive control. All the amplifications were performed with the same RNA samples and dilutions. Control reactions without reverse transcriptase showed no DNA contamination.
The transcript levels of the $\exp A, \exp C, \exp D$, and $\exp E$ promoters were, if present, very low in the mucR and mucRS mutants, therefore the possibility that MucR is not only necessary for $m u c S$ transcription but also activates exp genes transcription through other pathways cannot be excluded. To test this hypothesis, a cloned mucS gene was introduced into the $m u c R$ mutant, either under the control of its own promoter (pBG1140) or under the control of the strong nptII promoter (pBG1228). The introduction of pBG1140 had no effect on the colony morphology of the mucR mutant, which remained completely dry. However, when pBG1228 was introduced into the mucR mutant, colonies were mucoid, although at a lower level than in the wild type. Together with the RT-PCR results, these data indicate that $m u c R$ is a strict requirement for the transcription of $m u c S$ and that, in addition to a functional MucS, mucR is necessary for the full production of galactoglucan. If $m u c R$ has a role other than mucS transcriptional activation, it should influence $\exp$ genes transcription through another pathway.

\section{A palindromic, conserved sequence is located at the distal part of $\exp$ promoters.}

We have previously shown that the $\exp E$ promoter is highly expressed in EFB1 and that there is a 95\% reduction in expression in a mucS mutant strain (Martin et al. 2000). To identify putative regulatory regions within this promoter, we have analyzed the expression level from nested deletions of the promoter. To construct these deletions, nested forward primers and a common reverse primer were designed from the $\exp E$ promoter sequence and were used to amplify genomic DNA from EFB1. The PCR-resulting fragments were cloned in the pCR2.1 plasmid, sequenced, and directionally fused to a promoterless $l a c Z$ gene in plasmid pMP220, giving rise to the reporter constructs described in Table 2 . $\beta$-galactosidase activity assays were done in EFB1, EFB207, EFB208, and EFB209 backgrounds. As shown in Figure 4, the expression of the expE promoter was very high in strain EFB1, and there was a drastic decrease in activity in the EFB207 background. Deletion of up to 138 nucleotides in the $5^{\prime}$ region of the promoter (pRO1085 and pRO1086) did not have major effects on activity or regulation by MucS. However, a further deletion of only $30 \mathrm{bp}$ (pRO1069) resulted in a drastic reduction of activity in the EFB1 background to levels found in EFB207, indicating the loss of mucS regulation. These results strongly indicate that this 30-bp region must be important for MucS as a transcriptional activator of $\operatorname{expE}$. The lack of expression observed for any of the constructs tested in the EFB208 and EFB209 backgrounds is consistent with $m u c R$ playing an additional role in $\exp E$ transcription.

The 30-bp expE putative regulatory region was compared with the exp intergenic sequences using the Bestfit and Gap

Table 1. Primers and annealing temperatures used for reverse transcription-polymerase chain reaction of the exp transcripts

\begin{tabular}{|c|c|c|c|}
\hline $\begin{array}{l}\exp \\
\text { gene }\end{array}$ & $\begin{array}{c}\text { Primer } \\
\text { no. }\end{array}$ & Primer sequence $\left(5^{\prime}-3^{\prime}\right)$ & $\begin{array}{c}\text { Annealing } \\
\text { temperature }\left({ }^{\circ} \mathrm{C}\right)\end{array}$ \\
\hline \multirow[t]{2}{*}{$\exp A$} & 1 & ATCGCCGACGAAGCGAAG & \multirow[t]{2}{*}{55} \\
\hline & 2 & TGCGGGCACGTCGATCC & \\
\hline \multirow[t]{2}{*}{$\exp C$} & 3 & GATGCCGCCATCCATCG & \multirow[t]{2}{*}{55} \\
\hline & 4 & CGCCGCCTCGGGCAG & \\
\hline \multirow[t]{2}{*}{ mucS } & 5 & CCCGTAACGCGCGGC & \multirow[t]{2}{*}{53} \\
\hline & 6 & ATGGAGAGAGGGATGAAC & \\
\hline \multirow[t]{2}{*}{$\exp D$} & 7 & ACCGCTGATGCAACTGGAG & \multirow[t]{2}{*}{59} \\
\hline & 8 & ACGAAATAGGCGACATTGGAC & \\
\hline \multirow[t]{2}{*}{$\exp E$} & 9 & TGGCAGCGACTTCCTTCT & \multirow[t]{2}{*}{55} \\
\hline & 10 & CGACATTGCCTCCGACCTG & \\
\hline
\end{tabular}


programs (Genetic Computer Group, Madison, WI, U.S.A.). A region of $20 \mathrm{bp}$ within the $30 \mathrm{bp}$ was highly conserved in equivalent positions at the distal part of the putative $\exp A$ and $\operatorname{expD}$ promoters in EFB1 (Fig. 5) and Rm2011 (data not shown) but not in the distal part of the putative mucS promoter of both strains. In the case of $\exp C$, the intergenic region located upstream of the gene is very short and does not contain putative regulatory sequences. However, the same conserved region is found 31 nucleotides upstream of the $m u c S$ start codon and in the sense of the transcription of mucS and $\operatorname{expC}$ (Fig. 1). It partially overlaps the conserved region in the putative $\operatorname{expD}$ promoter. As mucS is not autoregulated and this conserved region is located very close to the mucS ATG codon, it is likely that the $\exp C$ promoter overlaps the mucS coding sequence, and therefore, the conserved region located just upstream of mucS corresponds to the $\exp C$ promoter.
The conserved regions contain a palindrome (Fig. 5, inverted arrows), although homology extends beyond it. Palindromes, such as these with an AT-rich axis, are typical binding sites for helix-turn-helix proteins such as MucS (Becker et al. 1997). The presence of this palindromic region in the putative $\exp A$, $\exp C, \exp D$, and $\operatorname{expE}$ promoters, and the fact that MucS is activating the transcription of the exp genes but not its own transcription, strongly indicates that it corresponds to the target site of the MucS protein. For this reason, we have termed the 20-bp region the mис box. These mис boxes do not correspond to the pho boxes described by Rüberg and associates (1999).

\section{A mutant unable to produce galactoglucan is affected in competitive nodulation.}

The biological significance of galactoglucan production is not well defined. Gonzalez and associates (1996) have shown

Table 2. Strains and plasmids used

\begin{tabular}{|c|c|c|}
\hline Strain or plasmid & Relevant characteristics & Source or reference \\
\hline \multicolumn{3}{|c|}{ Sinorhizobium meliloti } \\
\hline EFB1 & Wild type, $\mathrm{Sm}^{\mathrm{r}}$ & Lloret et al. 1995 \\
\hline EFB207 & EFB1 1 mucS & This study \\
\hline EFB208 & mисR::pK18mobsacB & Martin et al. 2000 \\
\hline EFB209 & EFB207 mисR::pK18mobsacB & This study \\
\hline \multicolumn{3}{|l|}{ Escherichia coli } \\
\hline DH5 $\alpha$ & $\operatorname{rec} A 1 \triangle(\operatorname{lac} Z Y A-\arg F)$ & Gibco, Rockville, MD, U.S.A. \\
\hline \multicolumn{3}{|l|}{ Plasmids } \\
\hline pRK2013 & $\mathrm{Km}^{\mathrm{r}}$ ColE1 replicon with PK2 tra genes & Figurski and Helinski 1979 \\
\hline pMP220 & $\mathrm{Tc}^{\mathrm{r}} m o b^{+} l a c Z \mathrm{IncP}$ & Spaink et al. 1987 \\
\hline pCR 2.1-TOPO & $\mathrm{Amp}^{\mathrm{r}} \mathrm{Km}^{\mathrm{r}}$ & Invitrogen, Carsbad, CA, U.S.A. \\
\hline pBluescript & $\mathrm{Amp}^{\mathrm{r}}$ phagemid M13 derivative $\mathrm{f} 1$ origin of replication & Stratagene, La Jolla, CA, U.S.A. \\
\hline pK18mobsacB & $\mathrm{Km}^{\mathrm{r}}$ pUC18 derivative $l a c Z$ mob site $s a c B$ & Schäfer et al. 1994 \\
\hline pLAFR3 & $\mathrm{Tc}^{\mathrm{r}}$ host range $\mathrm{P}$-group cloning vector, mob RK 2 cosmid & Staskawicz et al. 1987 \\
\hline pML122 & $\mathrm{Km}^{\mathrm{r}} \mathrm{RSF} 1010$ derivative expression vector & Labes et al. 1990 \\
\hline pBG1000 & pLAFR3 with $24 \mathrm{~kb}$ of the $\exp$ gene cluster of EFB1 & Lloret et al. 1998 \\
\hline pBG1140 & pLAFR3 with mucS gene & This study \\
\hline pBG1141 & pLAFR3 with $\Delta m u c S$ & This study \\
\hline pBG1228 & pML122 with mucS under the control of the vector Nm promoter & This study \\
\hline pRO1052 & $\begin{array}{l}\text { pMP } 220 \text { with } 346 \mathrm{bp} S p h \mathrm{I} \text { fragment containing the } \operatorname{expE} \text { promoter in sense } \\
\text { orientation of EFB } 1\end{array}$ & Lloret et al. 1998 \\
\hline pRO1069 & pRO1052 with a 142-bp deletion at the distal part of $\operatorname{expE}$ promoter & This study \\
\hline pRO1084 & pRO1052 with a 187-bp deletion at the distal part of $\operatorname{expE}$ promoter & This study \\
\hline pRO1985 & pRO1052 with a 92b-p deletion at the distal part of $\operatorname{expE}$ promoter & This study \\
\hline pRO1086 & pRO1052 with a 112-bp deletion at the distal part of $\operatorname{expE}$ promoter & This study \\
\hline
\end{tabular}

${ }^{a} \mathrm{Sm}^{\mathrm{r}}, \mathrm{Km}^{\mathrm{r}}, \mathrm{Tc}^{\mathrm{r}}$, and $\mathrm{Amp}^{\mathrm{r}}$ mean resistant to streptomycin, kanamycin, tetracycline, and ampicillin, respectively.

\section{EFB1 EFB207 EFB208 EFB209}

\begin{tabular}{|c|c|c|c|c|c|}
\hline pRO1052 & lacZ & 8387 & 450 & 65 & 74 \\
\hline pRO1085 & & 8510 & 2308 & n.d. & n.d. \\
\hline pRO1086 & & 6969 & 1330 & 209 & 190 \\
\hline pR01069 & & 1473 & 2362 & n.d. & n.d. \\
\hline pRO1084 & & 2472 & 1340 & n.d. & n.d. \\
\hline
\end{tabular}

Fig. 4. Transcription analysis of the expE promoter in strain EFB1 and mutant backgrounds; EFB1 (wild type), EFB207 (mucS ${ }^{-}$), EFB208 (mucR ${ }^{-}$), and EFB209 (mucS ${ }^{-}$mucR ${ }^{-}$). Nested deletions of the expE promoter were directionally fused to a promoterless lacZ gene in the pMP220 vector and introduced in the rhizobial strains by triparental mating. $\beta$-galactosidase activity was determined according to Miller (1972). n.d. $=$ Not determined. $\beta$-galactosidase activity of EFB1 was 24 units and of EFB1 (pMP220) was 133 units. 
that galactoglucan can replace succinoglycan in noninfecting exo mutants that do not produce succinoglycan. Besides, the $\operatorname{expR}$ mutation, which results in overexpression of the exp genes, has been shown to suppress the symbiotic defect of succinoglycan-deficient strains (Glazebrook and Walker 1989). However, when galactoglucan is produced under phosphatelimiting conditions, it does not allow the invasion of alfalfa nodules (Mendrygal and Gonzalez 2000).

The mutant strain EFB209 does not show expression of any of the $\exp$ promoters and should not produce any galactoglucan. We have used this strain to investigate the possible role of this exopolysaccharide in $S$. meliloti-alfalfa symbiosis. Competition assays between EFB1 and EFB209 were performed in Leonard jars, using perlite as the solid substrate, and in sterilized and nonsterilized soil microcosms in Riviera pots. As shown in Table 3, no differences in competitivity were observed in Leonard jar systems, with each strain forming approximately half of the analyzed nodules and with a small proportion of nodules formed by both strains simultaneously. However, when the experiments were performed in soil microcosms, the mutant strain EFB209 was clearly outcompeted by wild-type EFB1 and by the indigenous rhizobial population. It is interesting to note that the mutation of the mucR homologue ros $R$ in $R$. etli results in a noncompetitive mutant (Bittinger et al. 1997). No differences in the lack of EFB209 competitiveness were observed between sterilized and nonsterilized soil microcosms, suggesting that galactoglucan might play a role in adaptation to abiotic stress conditions. In this sense, Mendrygal and Gonzalez (2000) have suggested that exopolysaccharides may play a role in the transition between the soil and the plant

Table 3. Percentage of nodules formed by each strain in competition experiments

\begin{tabular}{lccc}
\hline & \multicolumn{3}{c}{ Substrate } \\
\cline { 2 - 4 } Strain & Perlite $^{\mathbf{a}}$ & Sterilized soil $^{\mathbf{b}}$ & Nonsterilized soil $^{\mathbf{c}}$ \\
\hline EFB1 & 49 & 91.4 & 33.3 \\
EFB209 & 44 & 8.6 & 0 \\
Other rhizobia & 0 & 0 & 66.7 \\
Co-occupancy & 7 & 0 & 0 \\
\hline${ }^{\mathrm{a}} n=100$ nodules. & & & \\
${ }^{\mathrm{b}} n=35$ nodules. & & \\
${ }^{\mathrm{c}} n=30$ nodules. & & \\
${ }^{\mathrm{d}}$ EFB1 and EFB209. & & &
\end{tabular}

environments. Because galactoglucan production is higher at low phosphate levels and the nodule is a high-phosphate environment, they assume that this exopolysaccharide is produced primarily while $S$. meliloti is in the soil and not inside the nodule.

Our results indicate that, in the $S$. meliloti strain EFB1, exp genes expression and, therefore, galactoglucan production is primarily regulated by $\mathrm{MucR}$, which is necessary for the transcription of the mucS gene and possibly implicated in the transcription of the other exp promoters. This is in contrast to its role in strain $\mathrm{Rm} 1021$, in which MucR confers negative regulation of exp genes transcription. It should be noted that the $R$. etli MucR homologue (RosR) regulates more than 50 genes (Bittinger and Handelsman 2000). The fact that no MucR-binding sites (Keller et al. 1995) have been found in the exp promoters (including $m u c S$ ) indicates that this regulation should be indirect. MucS, in turn, is a transcriptional activator of the $\exp A, \exp C, \exp D$, and $\exp E$ operons and probably binds to the conserved muc boxes present within the exp promoters.

\section{MATERIALS AND METHODS}

\section{Strains, plasmids, and culture conditions.}

The bacterial strains and plasmids used in this study are listed in Table 2. S. meliloti was grown at $28^{\circ} \mathrm{C}$ in TY medium (Beringer 1974). Escherichia coli strains were grown at $3^{\circ} \mathrm{C}$ in Luria Bertani (LB) medium. Antibiotics were added at the following concentrations when required: $200 \mu \mathrm{g}$ of streptomycin per $\mathrm{ml}, 10 \mu \mathrm{g}$ of tetracycline per $\mathrm{ml}, 20 \mu \mathrm{g}$ of kanamycin per $\mathrm{ml}$, and $100 \mu \mathrm{g}$ of ampicillin per $\mathrm{ml}$.

\section{DNA techniques.}

Standard techniques for subcloning procedures, plasmid preparations, and agarose gel electrophoresis were used as described by Sambrook and associates (1989). Southern blot hybridizations were performed with a nonradiactive detection kit, and a chemiluminiscence method was used to detect hybridization bands according to the manufacturer's instructions (Roche Diagnostics, Barcelona, Spain). DNA sequencing was done by the chain termination method using the DyeDeoxy terminator cycle sequencing kit protocol as described by the manufacturer (Applied Biosystems, Madrid, Spain). Homology search and sequence analysis were performed using software from the Genetics Computer Group (Madison, WI, U.S.A.).

\section{Distance to ATG}

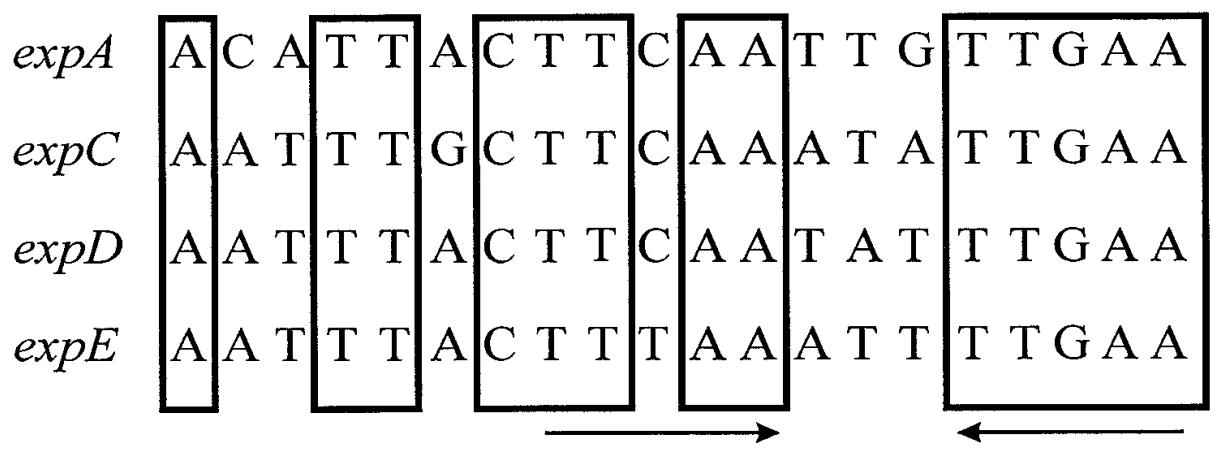

Fig. 5. Alignment of the conserved region found in the distal part of the putative $\exp A, \exp C, \exp D$, and $\operatorname{expE}$ promoters of Sinorhizobium meliloti EFB1. Residues identical in the four promoters are boxed. Inverted arrows indicate a palindrome found within the conserved region. The distance in nucleotides to the ATG of the first open reading frame in each operon is indicated. The accession number for the sequence of the $S$. meliloti EFB1 exp region is Y08703. 


\section{RT-PCR assays.}

Total RNA from strains EFB1, EFB207, EFB208, and EFB209 was extracted following the SV RNA isolation system from Promega (Madison, WI, U.S.A.). For subsequent RTPCR, forward- and reverse-strand primers were designed from $\exp A, \exp C, \exp D, \exp E$, and $m u c S$ genes and are described in Table 1 . The reactions were done following the RT-PCR system instructions from Promega. RT reaction was done at $48^{\circ} \mathrm{C}$ for $45 \mathrm{~min}$, followed by a denaturation step and 40 PCR cycles. The appropriate band length was checked by agarose gel electrophoresis. Negative controls were done without RT reaction to detect putative DNA contamination of the RNA samples.

Deletions of the $\exp E$ promoter and $\beta$-galactosidase assay.

PCR deletions were done using a common, reverse-strand primer, which contains an SphI site, 5' GCATGCGTCATCGGCAC $3^{\prime}$. The forward-strand primer used to amplify the complete promoter was $5^{\prime}$ CCCGCGGAAGTGCTGAT 3'. The nested forward primers used to amplify the different primer deletions were $5^{\prime}$ GCATCAATTGGCAATTTACTT 3', 5' TTAAATTTTGAACCGGTTTGC $3^{\prime}$, and $5^{\prime}$ TCCGCAAGAATAACTCCTAAAA 3'. The PCR products were cloned in the pCR-2.1 vector (Invitrogen, Carsbad, CA, U.S.A.) and were digested with EcoRI and SphI to be directionally cloned in pMP220 in the sense of the transcription of the lacZ gene (Spaink et al. 1987). The reporter constructs (Table 2) were introduced in the rhizobial strains by triparental mating using the helper plasmid pRK2013. Reporter strains were grown on TY, and $\beta$-galactosidase activity was assayed after $18 \mathrm{~h}$ of growth. $\beta$-galactosidase units were calculated as described by Miller (1972).

\section{Competition studies.}

Competition experiments were performed in Leonard jar systems and soil microcosms. Alfalfa seeds were surface-sterilized with bleach and $70 \%$ ethanol, rinsed with sterile water, and germinated on agar plates. Seedlings were placed in Leonard jars with perlite and Riviera pots with either autoclaved or nonautoclaved sieved soil and inoculated with approximately $10^{8}$ cells from exponentially growing TY cultures of each strain. Inoculated plants were grown for 4 weeks in a growth cabinet with a photoperiod of $16 \mathrm{~h}$ of light at $25^{\circ} \mathrm{C}$ and $8 \mathrm{~h}$ of dark at $18^{\circ} \mathrm{C}$. Nodules were excised, surface-sterilized with $70 \%$ ethanol, and crushed in liquid TY medium. Crushed nodules were inoculated on TY plates containing the appropriate antibiotics to differentiate the strain forming the nodule. Nodule occupancy was also assessed by the morphology of the colonies.

\section{ACKNOWLEDGMENTS}

We wish to thank J. A. Downie for his helpful discussions. R. I. Oruezabal was supported by a predoctoral fellowship from the Basque Country Government. The research was supported by Dirección General de Enseñanza Superior e Investigación Científica grant PB98-0114-CO2-01.

\section{LITERATURE CITED}

Astete, S. G., and Leigh, J. A. 1996. mucS, a gene involved in activation of galactoglucan (EPS II) synthesis gene expression in Rhizobium meliloti. Mol. Plant-Microbe Interact. 9:395-400.

Battisti, L., Lara, J. C., and Leigh, J. A. 1992. Specific oligosaccharide form of the Rhizobium meliloti exopolysaccharide promotes nodule invasion in alfalfa. Proc. Natl. Acad. Sci. U.S.A. 89:5625-5629.
Becker, A., Rüberg, S., Küster, H., Roxlau, A. A., Keller, M., Ivashina, T. Cheng, H., Walker, G. C., and Pühler, A. 1997. A 32-kilobase $\exp$ gene cluster of Rhizobium meliloti directing the biosynthesis of galactoglucan: Genetic organization, properties of the encoded gene products. J. Bacteriol. 179:1375-1384.

Beringer, J. E. 1974. R factor transfer in Rhizobium leguminosarum. J. Gen. Microbiol. 84:188-198.

Bertram-Drogatz, P. A., Quester, Y., Becker, A., and Puhler, A. 1998. The Sinorhizobium meliloti MucR protein, which is essential for the production of high-molecular-weight succinoglycan exopolysaccharide, binds to short DNA regions upstream of exoH and exoY. Mol. Gen. Genet. 257:433-441.

Bittinger, M. A., and Handelsman, J. 2000. Identification of genes in the RosR regulon of Rhizobium etli. J. Bacteriol. 182:1706-1713.

Bittinger, M. A., Milner, J. L., Saville, B. J., and Handelsman, J. 1997. rosR, a determinant of nodulation competitiveness in Rhizobium etli. Mol. Plant-Microbe Interact. 10:180-186.

Cooley, M. B., D'Souza, M. R., and Kado, C. Y. 1991. The virC and virD operons of the Agrobacterium Ti plasmid are regulated by the rosAT chromosomal gene: Analysis of the cloned rosAT gene. J. Bacteriol. 173:2608-2616.

Figurski, D. H., and Helinski, D. R. 1979. Replication of an origincontaining derivative of plasmid RK2 dependent on a plasmid function provided in trans. Proc. Natl. Acad. Sci. U.S.A. 76:1648-1652.

Glazebrook, J., and Walker, G. C. 1989. A novel exopolysaccharide can function in place of the calcofluor-binding exopolysaccharide in nodulation of alfalfa by Rhizobium meliloti. Cell 56:661-672.

Gonzalez, J. E., Reuhs, B. L., and Walker, G. C. 1996. Low molecular weight EPS II of Rhizobium meliloti allows nodule invasion in Medicago sativa. Proc. Natl. Acad. Sci. U.S.A. 93:8636-8641.

Keller, M., Roxlau, A., Weng, W. M., Schmidt, M., Quandt, J., Niehaus, K., Jording, D., Arnold, W., and Pühler, A. 1995. Molecular analysis of the Rhizobium meliloti mucR gene regulating the biosynthesis of the exopolysaccharides succinoglycan and galactoglucan. Mol. PlantMicrobe Interact. 8:267-277.

Labes, M., Pühler, A., and Simon, R. 1990. A new family of RSF1010derived expression and lac-fusion broad-host-range vectors for gramnegative bacteria. Gene 89:37-46.

Leigh, J. A., and Walker, G. C. 1994. Exopolysaccharides of Rhizobium: Synthesis, regulation, symbiotic function. Trends Genet. 10:63-67.

Lloret, J., Bolaños, L., Lucas, M. M., Peart, J., Brewin, N. J., Bonilla, I. and Rivilla, R. 1995. Ionic and osmotic pressure induce different alterations in the lipopolysaccharide of a Rhizobium meliloti strain Appl. Environ. Microbiol. 61:3701-3704.

Lloret, J., Wulff, B. B., Rubio, J. M., Downie, J. A., Bonilla, I., and Rivilla, R. 1998. Exopolysaccharide II production is regulated by salt in the halotolerant strain Rhizobium meliloti EFB1. Appl. Environ. Microbiol. 64:1024-1028.

Martín, M., Lloret, J., Sánchez-Contreras, M., Bonilla, I., and Rivilla, R. 2000. MucR is necessary for galactoglucan production in Sinorhizobium meliloti EFB1. Mol. Plant-Microbe Interact. 13:129-135.

Mendrygal, K. E., and Gonzalez, J. E. 2000. Environmental regulation of exopolysaccharide production in Sinorhizobium meliloti. J. Bacteriol. 182:599-606

Miller, J. H. 1972. Experiments in Molecular Genetics. Cold Spring Harbor Laboratory Press, Cold Spring Harbor, NY, U.S.A.

Rüberg, S., Pühler, A., and Becker, A. 1999. Biosynthesis of the exopolysaccharide galactoglucan in Sinorhizobium meliloti is subject to a complex control by the phosphate-dependent regulator PhoB and the proteins ExpG and MucR. Microbiology 145:603-611.

Sambrook, J., Fritsch, E. F., and Maniatis, T. 1989. Molecular Cloning: A Laboratory Manual, 2nd ed. Cold Spring Harbor Laboratory Press, Cold Spring Harbor, NY, U.S.A.

Schäfer, A., Tauch, A., Jäger, W., Kalinowski, J., Thierbach, G., and Pühler, A. 1994. Small mobilizable multipurpose cloning vectors derived from the Escherichia coli plasmids pK18 and pK19: Selection of defined delections in the chromosome of Corynebacterium glutamicum. Gene 145:69-73.

Spaink, H. P., Okker, R. J. H., Wijffelman, C. A., Pees, E., and Lugtenberg, B. J. J. 1987. Promoters in the nodulation region of the Rhizobium leguminosarum Sym plasmid pRL1JI. Plant Mol. Biol. 9:27-39.

Staskawicz, B., Dahlbeck, D., Keen, N., and Napoli, C. 1987. Molecular characterization of cloned avirulence genes from race 0 and race 1 of Pseudomonas syringae pv. glycinea. J. Bacteriol. 169:5789-5794. 